\title{
Glucose Oxidase-immobilized Glass Disks for Imaging of D-Glucose in Acute Brain Slices
}

\author{
Nobukatsu Moridera, Miki Yamamoto, Wataru OKumura, and Masao Sugawara ${ }^{\dagger}$ \\ Department of Chemistry, College of Humanities and Sciences, Nihon University, \\ Sakurajousui, Setagaya, Tokyo 156-8550, Japan
}

\begin{abstract}
A biotinylated glucose oxidase (bGOD)-immobilized glass disk was prepared for visualizing D-glucose fluxes in acute brain slices. A mouse hippocampal slice was placed on the bGOD disk and stimulated with a stimulant solution containing horseradish peroxidase (HRP) and a substrate DA-64, followed by capturing digital images of Bindschedler's Green (BG), an oxidized form of DA-64, with a CCD camera. The bGOD membranes responded proportionally to Dglucose, ranging from 2.0 to $5.0 \mathrm{mM}$. Sucrose, GABA, L-glutamic acid, L-aspartic acid, glycine, acetylcholine and Lascorbic acid at $10 \mathrm{mM}$ did not cause any responses. The D-glucose fluxes in mouse hippocampal slices stimulated by a hypoxia solution were neuronal region-dependent, i.e., dentate gyrus (DG), cornu ammonis 1 (CA1) and cornu ammonis 3 (CA3), while those stimulated by $\mathrm{KCl}$ was independent of the neuronal regions. The response of bGOD disks is discussed in terms of the principle, concentration dependence and selectivity.
\end{abstract}

(Received August 23, 2006; Accepted November 6, 2006; Published January 10, 2007)

\section{Introduction}

Glucose metabolism is the major pathway of energy production in the mature brain. ${ }^{1}$ The relationship between neuronal activity, especially glutamatergic neuronal acitivity, ${ }^{2,3}$ and energy consumption has attracted attention concerning the energetic basis of brain activity. For the in vivo mapping of brain activity, functional magnetic resonance imaging (fMRI), positron-emission tomography (PET) and magnetic resonance spectroscopy (MRS) are the dominant methods.4-6 On the other hand, for in vitro imaging of the spatial and temporal distribution of glucose in brain slices, which are one of the most common models of the brain, a few methods have been reported, including autoradiography with radio-labeled glucose and related compounds, ${ }^{7-10}{ }^{31} \mathrm{P}$ NMR, ${ }^{11}$ and the measurements of intrinsic optical signals. ${ }^{12,13}$ Although these methods have been utilized for elucidating glucose metabolisms in brain slices or cultured slices, the use of radio-labeled compounds is hampered by ease of handling and safety. The intrinsic optical signal measures any cell swelling, i.e., volume changes, rather than D-glucose, itself. ${ }^{14,15}$ In the present paper, extending our previous approach ${ }^{15,16}$ to glucose systems, we describe an enzyme-based imaging method for D-glucose in acute brain slices. Biotinylated glucose oxidase (bGOD) is immobilized on a glass disk by the avidinbiotin technique. Brain slices are placed on the bGOD disks, followed by stimulation with a solution containing a stimulant, horse radish peroxidase (HRP) and an oxidation dye DA-64. Glucose triggers an enzyme reaction and oxidizes the substrate DA-64 to Bindschedler's Green (BG) according to

$$
\text { D-glucose }+\mathrm{O}_{2}+\mathrm{H}_{2} \mathrm{O} \stackrel{\text { bGOD }}{\longrightarrow} \text { gluconic acid }+\mathrm{H}_{2} \mathrm{O}_{2}
$$

$\dagger$ To whom correspondence should be addressed.

E-mail: sugawara@chs.nihon-u.ac.jp

$$
\mathrm{H}_{2} \mathrm{O}_{2}+2 \mathrm{H}^{+}+\text {DA- } 64 \stackrel{\mathrm{HRP}}{\longrightarrow} \text { Bindschedler's Green }+2 \mathrm{H}_{2} \mathrm{O}
$$

By applying a differential image analysis, glucose fluxes at various neuronal regions of mouse hippocampal slices are imaged. The response of the bGOD disks to D-glucose is discussed in terms of the principle, concentration dependence and selectivity. The bGOD disks are then applied to in vitro imaging of D-glucose fluxes in acute mouse hippocampal slices under a chemical stimulation. Since bGOD is first immobilized on the disk, the diffusion of bGOD into a brain slice is avoided. Consequently, chemical perturbation of the system will be minimized.

\section{Experimental}

\section{Reagents and chemicals}

Bitoinylated glucose oxidase (bGOD) and rhodamine avidin D were obtained from Vector Laboratories (Burlingame, CA, USA). Horseradish peroxidase (HRP), D-glucose, 2-deoxy-Dglucose, avidin and $N$-(carboxymethylaminocarbonyl)-4,4'bis(dimethylamino)diphenylamine sodium salt (DA-64) were obtained from Wako Pure Chemicals Co. (Osaka, Japan). A hetero-bifunctional reagent, $N$-(11-maleimidoundecanoyloxy)succinimide (KMUS) was obtained from Dojindo Laboratories (Kumamoto, Japan). Anti-biotin glucose oxidase was obtained from Rockland Immunochemical Inc. (Gilbertsville, PA, USA) and biocytin-oregon green was from Invitrogen Co. (Carlsbad, CA, USA). 3-Mercaptopropyltrimethoxysilane (MTS, >99.9\%) was obtained from Shin-Etsu Chemical (Tokyo, Japan). LGlutamic acid, L-aspartic acid, glycine, acetylcholine, $\gamma$ aminobutyric acid (GABA), dopamine, sucrose and L-ascorbic acid were purchased from Wako Pure Chemicals Co. Other chemicals used were all of analytical reagent grade.

Micro glass cover disks (18 $\mathrm{mm}$ in diameter, $0.17-0.25 \mathrm{~mm}$ in thickness) were obtained from Matsunami Glass Industries 
(Tokyo, Japan). Milli-Q water (Millipore reagent water system, Bedford, MA) was used throughout the experiments.

A recovery solution ( $\mathrm{pH} 7.4$ ) contained $124 \mathrm{mM} \mathrm{NaCl}, 3.0$ $\mathrm{mM} \mathrm{KCl}, 26 \mathrm{mM} \mathrm{NaHCO}, 1.25 \mathrm{mM} \mathrm{NaH} \mathrm{PO}_{4}, 1.3 \mathrm{mM}$ $\mathrm{MgSO}_{4}, 2.0 \mathrm{mM} \mathrm{CaCl}_{2}$ and $15 \mathrm{mM}$ D-glucose, saturated with a $95 \% \mathrm{O}_{2}-5 \% \mathrm{CO}_{2}$ gas mixture. A glucose-free recovery solution was prepared by replacing D-glucose in the recovery solution with an equimolar amount of 2-deoxy-D-glucose. A glucosefree hypoxia solution was prepared by bubbling a glucose-free recovery solution containing $5.3 \mathrm{mM} \mathrm{DA}-64$ and $0.6 \mathrm{mg} / \mathrm{ml}$ $\mathrm{HRP}$ with a $95 \% \mathrm{~N}_{2}-5 \% \mathrm{CO}_{2}$ gas mixture. A KCl-stimulant solution contained $0.10 \mathrm{M} \mathrm{KCl}, 5.3 \mathrm{mM}$ DA-64 and $0.6 \mathrm{mg} / \mathrm{ml}$ HRP in a glucose-free recovery solution. A standard D-glucose solution was prepared by dissolving D-glucose in Milli-Q water. A HRP solution contained a $0.1 \mathrm{M} \mathrm{NaH}_{2} \mathrm{PO}_{4} / \mathrm{NaOH}(\mathrm{pH} 7.0)$, $5.3 \mathrm{mM}$ DA-64 and $0.6 \mathrm{mg} / \mathrm{ml} \mathrm{HRP}$.

Immobilization of glucose oxidase on an avidin-modified glass disk Biotinylated glucose oxidase (bGOD)-immobilized disks were prepared by immobilizing bGOD on avidin-modified glass disks. First, cover disks (diameter $18 \mathrm{~mm}$ ) were washed in $1 \mathrm{M}$ $\mathrm{NaOH}$ overnight, thoroughly washed with Milli-Q water and dried at $70^{\circ} \mathrm{C}$. One-side surface of a cover disk was silanized by treating with $100 \mu \mathrm{l}$ of $50 \mathrm{v} / \mathrm{v} \%$ MTS in toluene for $1 \mathrm{~h}$ at room temperature. After washing with toluene and evaporating it, the disk was treated with $80 \mu \mathrm{l}$ of $2 \mathrm{mM}$ KMUS in DMSO (anhydrous) for $1 \mathrm{~h}$, followed by incubation with a $150-\mu \mathrm{l}$ portion of $0.10 \mathrm{mg} / \mathrm{ml}$ avidin in a $0.1 \mathrm{M} \mathrm{NaH}_{2} \mathrm{PO}_{4} / \mathrm{NaOH}$ buffer solution ( $\mathrm{pH}$ 7.0) (a phosphate solution) at room temperature for $1 \mathrm{~h}$. Finally, the disk was treated with $150 \mu \mathrm{l}$ of $0.5 \mathrm{M}$ ethanolamine $(\mathrm{pH}$ 8.4) for $30 \mathrm{~min}$ to deactivate remaining KMUS sites. The disks are hereafter called avidin disks. For immobilizing bGOD via the avidin-biotin reaction, the avidin disks were incubated with $150 \mu \mathrm{l}$ of a $0.15 \mathrm{M} \mathrm{NaCl}$ solution containing $25 \mathrm{mM} \mathrm{NaH} \mathrm{PO}_{4} / \mathrm{NaOH}(\mathrm{pH} 7.4)$ and $0.10 \mathrm{mg} / \mathrm{ml}$ bGOD for $1 \mathrm{~h}$. The disks were washed with Milli-Q water and stored in the dark at $4^{\circ} \mathrm{C}$ until use.

Fluorometric images of an avidin disk and a bGOD disk were obtained with a FluorImager 595 (Molecular Dynamics, Sunnyvale, CA, USA). An avidin disk was treated with $100 \mu \mathrm{l}$

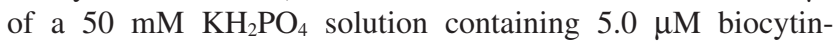
oregon green. The fluorescence image of the avidin disk showed the fluorescence of biocytin-oregon green (ex. $488 \mathrm{~nm}$ and em. $514 \mathrm{~nm}$ ) over the whole area of the avidin disk (image A in Fig. 1), indicating that avidin was homogeneously immobilized on the disk. Similarly, a bGOD disk was incubated with $200 \mu \mathrm{l}$ of a $0.15 \mathrm{M} \mathrm{NaCl}$ solution containing 20 $\mathrm{mM} \mathrm{KH} \mathrm{PO}_{4} / \mathrm{NaOH}(\mathrm{pH} 7.2)$ and $0.1 \mathrm{mg} / \mathrm{ml}$ biotinylated antiGOD, followed by washing with Milli-Q water. The disk was then incubated with $100 \mu \mathrm{l}$ of a $10 \mathrm{mM}$ HEPES/NaOH solution (pH 7.4) containing $0.1 \mathrm{mg} / \mathrm{ml}$ rhodamine avidin D. A biotin disk was also prepared as a reference disk by incubating an

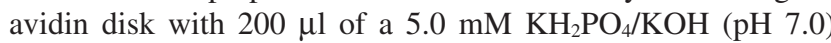
solution containing $0.1 \mathrm{mg} / \mathrm{ml}$ biotin. The fluorescence intensity (ex. $514 \mathrm{~nm}$ and em. $590 \mathrm{~nm}$ ) for the bGOD disk was 4-times larger than that for the biotin disk (image B in Fig. 1), demonstrating that bGOD was successfully immobilized on the avidin disk.

\section{Imaging with a standard D-glucose solution}

To mimic a brain slice, a piece of a square paper $(1 \times 1 \mathrm{~cm}$, thickness $5 \mu \mathrm{m}$ ) cut from a cellulose filter paper (Advantec, No. 2) was placed on a bGOD disk. The disk was set on a slide glass on the stage of a stereomicroscope ( $\mathrm{SZH}$, Olympus, Tokyo, Japan). A $40-\mu$ l portion of a HRP solution was placed

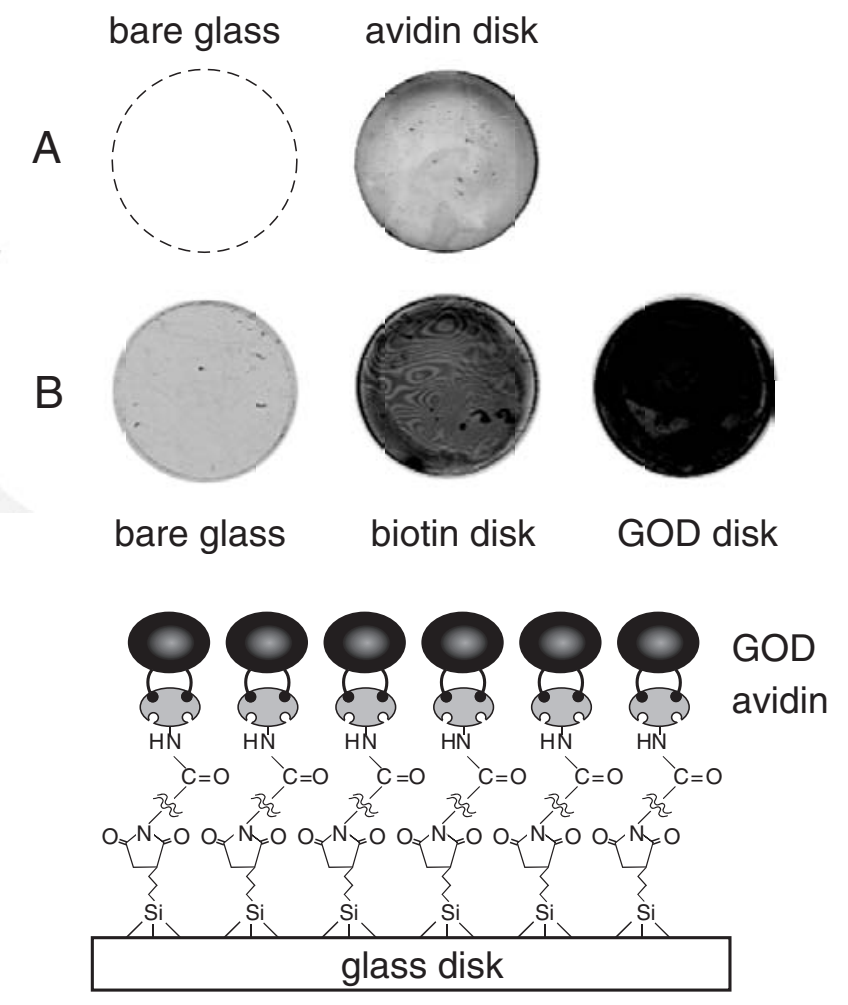

Fig. 1 Fluorometric characterization of avidin and bGOD disks. (A) Fluorescence images of an avidin-modified disk and a bare glass disk. The disks were treated with a $50 \mathrm{mM} \mathrm{KH}_{2} \mathrm{PO}_{4} / \mathrm{NaOH}(\mathrm{pH} 7.2)$ solution containing $5 \mu \mathrm{M}$ biocytin oregon green. Ex. $488 \mathrm{~nm}$ and em. $514 \mathrm{~nm}$. (B) Fluorometric images of bGOD and biotin disks. The disks were treated with a $0.15 \mathrm{M} \mathrm{NaCl}$ solution containing $20 \mathrm{mM}$ $\mathrm{KH}_{2} \mathrm{PO}_{4} / \mathrm{NaOH}(\mathrm{pH} 7.2)$ and $0.10 \mathrm{mg} / \mathrm{ml}$ biotinylated anti-glucose oxidase for $1 \mathrm{~h}$, and then with a HEPES/NaOH $(\mathrm{pH}$ 7.4) solution containing $0.10 \mathrm{mg} / \mathrm{ml}$ rhodamine avidin $\mathrm{D}$. The biotin disk was prepared by treating an avidin disk with a $5 \mathrm{mM} \mathrm{KH}_{2} \mathrm{PO}_{4} / \mathrm{KOH}(\mathrm{pH}$ 7.0) solution containing $0.10 \mathrm{mg} / \mathrm{ml}$ biotin. Ex. $514 \mathrm{~nm}$ and em. 590 $\mathrm{nm}$.

on the filter paper. Capturing digital images was performed every $10 \mathrm{~s}$ with a CCD camera (Coolpix 990, Nikon, Tokyo, Japan). After a lapse of $1 \mathrm{~min}$, a $40-\mu$ l portion of a D-glucose solution was added. The JPEG images taken by the CCD camera were converted into TIFF ones, which were opened with Aquacosmos version 2.5.0.0 (Hamamatsu Photonics, Hamamatsu, Japan).

\section{Slope analysis}

The obtained digital image was first divided into small regions, named as regions of interest (ROIs). A region of interest for its time-course analysis was defined as the area of the filter paper (vide supra), except for its edge. Next, the mean intensity of the red component, $R(t)$, over the ROI was evaluated and plotted against time, $t$, yielding a plot of $R(t) \mathrm{vs.} t$. For the $R(t) v s$. $t$ plot, a regression line was prepared in the time range from $(t-\Delta t)$ to $(t+\Delta t)$ based on the least-squares method, and the slope of the regression line was calculated. With differential parameters of $\Delta t=20,40,60$ and $80 \mathrm{~s}$, slope analyses were applied to the identical image. The value of $\Delta t=$ 40 was found to be the most appropriate; with other parameters, the slope analysis gave large noise or earlier responses. The slope of the $R(t) v$ s. $t$ plot has dimensions of $\mathrm{mol} \mathrm{min}^{-1} \mathrm{~cm}^{-2}$ and is a measure of a D-glucose flux at each measuring point (ROI). 
(a)

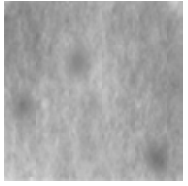

$0 \min$

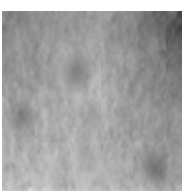

$3 \min$

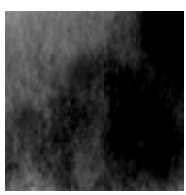

$6 \min$

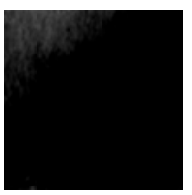

$9 \min$

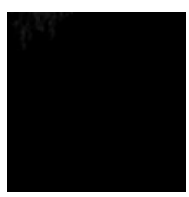

$12 \min$ (b)

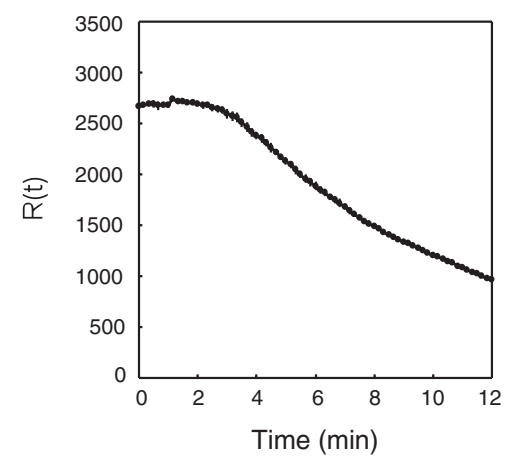

(c)

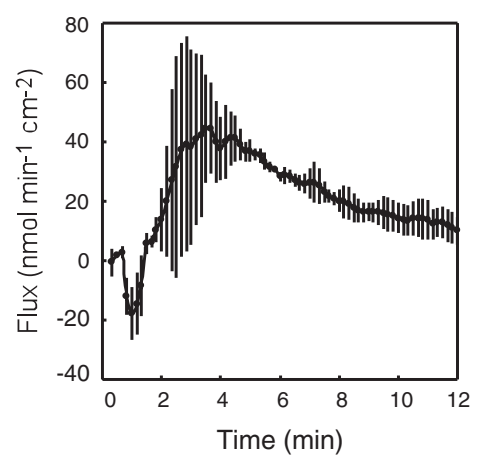

Fig. 2 Response of a bGOD membrane to D-glucose. (a) Time course of BG formation, (b) a plot of $R(t) v s . t$ and (c) the result of slope analyses. A $99-\mu \mathrm{l}$ portion of a $0.10 \mathrm{M}$ phosphate solution containing $5.3 \mathrm{mM}$ DA- 64 and $0.6 \mathrm{mg} / \mathrm{ml}$ HRP was placed. After $1 \mathrm{~min}$, a 1.0- $\mu \mathrm{l}$ portion of $0.30 \mathrm{M}$ D-glucose in Milli-Q water was injected. The measurements were made at $32 \pm 2^{\circ} \mathrm{C}$. The average of three measurements was plotted as the mean \pm standard deviation.

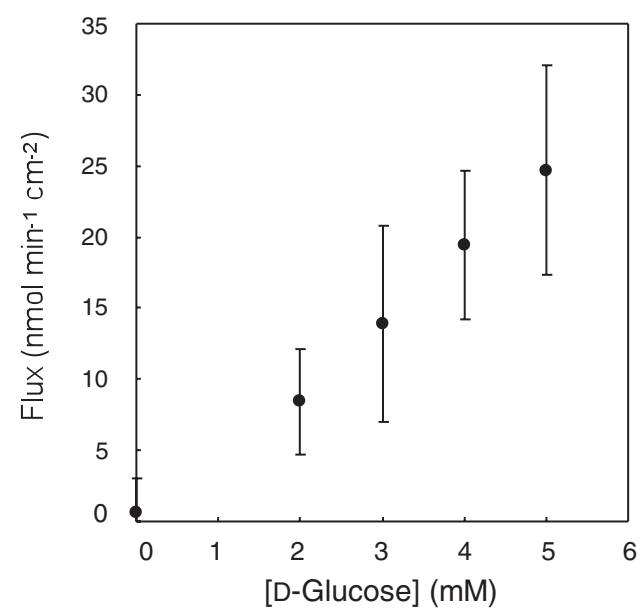

Fig. 3 Response of bGOD membranes to different concentration of D-glucose. A $99-\mu 1$ portion of a $0.10 \mathrm{M}$ phosphate solution containing $5.3 \mathrm{mM}$ DA-64 and $0.6 \mathrm{mg} / \mathrm{ml} \mathrm{HRP}$ was placed. After 1 min, a $1.0-\mu 1$ portion of a different concentration of D-glucose in Milli-Q water was injected. The stimulation was started at $t=0$. The magnitude of the flux $100 \mathrm{~s}$ after the addition of D-glucose in Milli-Q water was plotted. The measurements were made at $32 \pm 2^{\circ} \mathrm{C}$. The average of three measurements was plotted.

\section{Preparation of acute brain slices and D-glucose imaging}

Adult male ddY mice were decapitated under ether anesthesia. Coronal slices (thickness $300 \mu \mathrm{m}$ ) were cut using a microslicer (Dosaka DTK-1000, Kyoto, Japan) on an ice bath. The slices were incubated for $1 \mathrm{~h}$ in a recovery solution at $32^{\circ} \mathrm{C}$, and held at room temperature until use. A hippocampal slice was spooned from a recovery solution and washed twice with a glucose-free recovery solution. Then, the slice was transferred onto a bGOD disk. The excess solution on the slice was

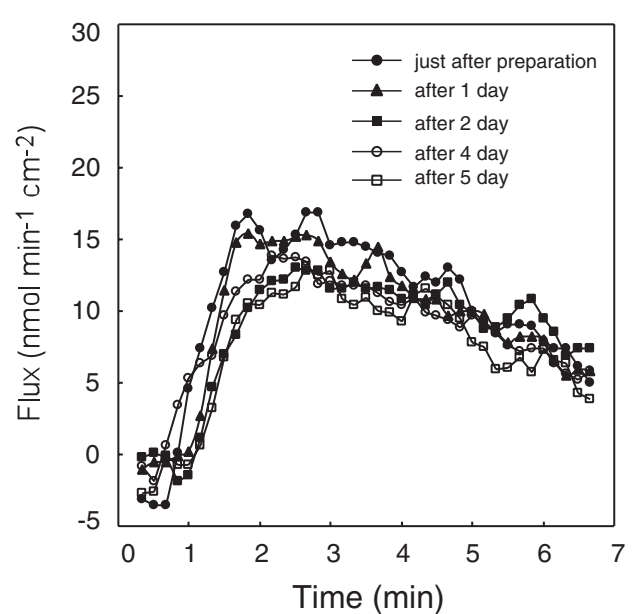

Fig. 4 Activity of immobilized bGOD after keeping in air at $4^{\circ} \mathrm{C}$. A $99-\mu$ l portion of a $0.10 \mathrm{M}$ phosphate solution containing $5.3 \mathrm{mM}$ DA- 64 and $0.6 \mathrm{mg} / \mathrm{ml} \mathrm{HRP}$ was placed. After $1 \mathrm{~min}$, a $1.0-\mu 1$ portion of $500 \mathrm{mM}$ D-glucose in Milli-Q water was injected.

removed by sucking up with a Kimwipe paper until the slice remained wet. The bGOD disk was set on a slide glass on the stage of a stereomicroscope. A $50-\mu$ l portion of a stimulant solution was placed on the slice, and taking bright-field photomicrographs with a CCD camera was started and repeated every $10 \mathrm{~s}$. All of the measurements were made at $32 \pm 2^{\circ} \mathrm{C}$.

TIFF images for the slice were divided into 14456 ROIs. The mean intensity of $R(t)$ was calculated for each ROI, yielding a plot of $R(t) v s . t$ and applied to a slope analysis. For preparing two dimensional images for neuronal regions, the size of each ROI was $5 \times 5$ pixels and each circular ROI had a size of $35 \times$ 35 pixels. 
(a) $D G$
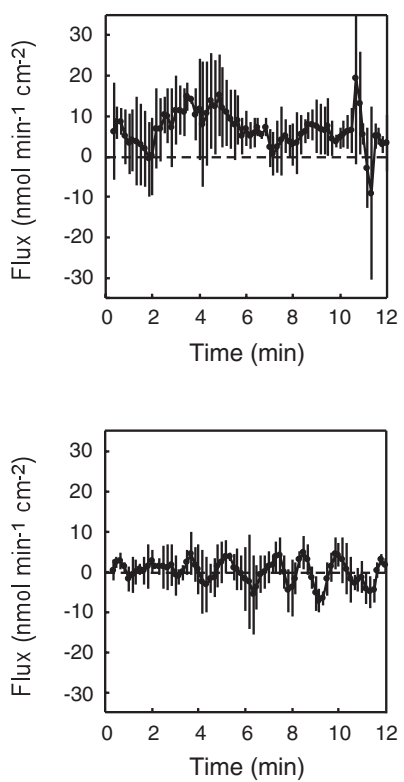

(b) CA1
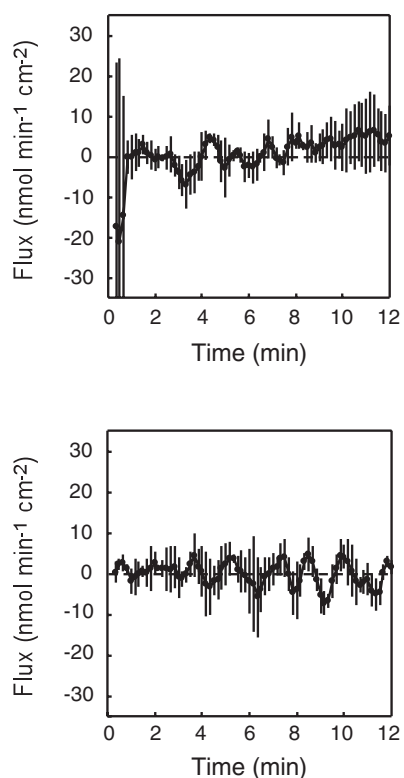

(c) $\mathrm{CA} 3$
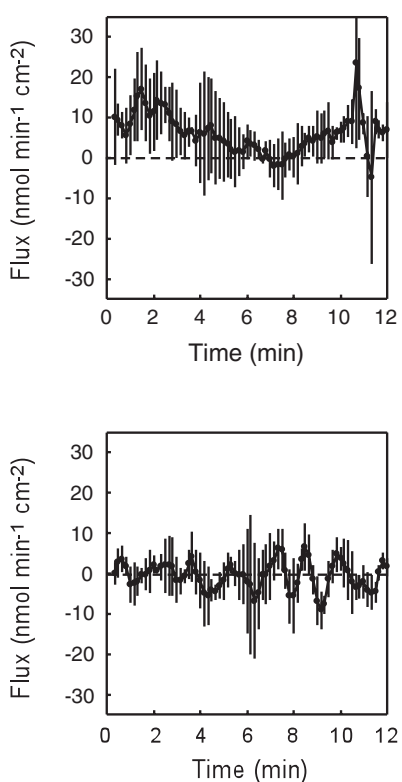

Fig. 5 Imaging of D-glucose fluxes in an acute mouse hippocampal slice under (A) hypoxia and (B) control. The slice was washed three times with a glucose-free recovery solution and stimulated with a 50- $\mu 1$ portion of (A) a hypoxia solution and (B) glucose-free recovery solution. The stimulation was started at $t=$ 0 . The measurements were done at $32 \pm 2{ }^{\circ} \mathrm{C}$. The average of three measurements was plotted.

\section{Results and Discussion}

Time-resolved images with a standard D-glucose solution

The enzymatic activity of bGOD immobilized on avidin disks was examined by measuring the response of a bGOD disk to Dglucose, i.e., the color development of BG. The consumption of D-glucose by bGOD at the surface of a bGOD disk has a stoichiometric relation with the generation of hydrogen peroxide, which triggers the formation of BG in the presence of HRP. A 50- $\mu$ l portion of a HRP solution (vide supra) was placed on a filter paper set on a bGOD disk. After 1 min, a 50$\mu 1$ portion of a $6.0 \mathrm{mM}$ D-glucose solution was added. Figure 2a shows the time course of color development of BG formed by the enzyme reaction in response to D-glucose. A deepening of green color with time is seen as enhanced dark images. The mean intensity over the whole area was evaluated, yielding a plot of $R(t) v s$. $t$ (Fig. 2b). The intensity of $R(t)$ decreased with time, corresponding to an increase in the amount of BG (absorption maximum $727 \mathrm{~nm}$ ). The result of the slope analysis (Fig. 2c) showed that the flux increased gradually, and reached the maximum after $2 \mathrm{~min}$, followed by a gradual decrease in the flux. The initial rise in the flux may correspond to the formation of a diffusion layer by the consumption of D-glucose at the membrane surface. After a lapse of time, the diffusion layer extended into the bulk of the solution, causing a gradual decrease in the D-glucose flux. When the filter paper was omitted, the similar flux-time profile was observed, and the time required to reach the maximum became shorter, i.e., about 1 min (data not shown).

The response of the bGOD disks to different concentrations of D-glucose in the range from 2.0 to $5.0 \mathrm{mM}$ is shown in Fig. 3. First, a 99- $\mu$ l portion of a HRP solution was placed on each bGOD disk, and after $1 \mathrm{~min}$, a $1.0-\mu \mathrm{l}$ portion of a known concentration of D-glucose in Milli-Q water was added. The magnitude of the flux $100 \mathrm{~s}$ after the addition of D-glucose was plotted against the initial concentration of D-glucose. The flux increased in proportion to the initial concentration of D-glucose, ranging from 2.0 to $5.0 \mathrm{mM}$. Two-dimensional visual images of the D-glucose fluxes (vide infra) could be obtained based on the calibration graph.

The selectivity of the bGOD disk to D-glucose over potential interfering compounds was investigated by placing a HRP solution containing each interfering compound on the bGOD disks. The responses 4 min after the injections of sucrose, $\gamma$ aminobutyric acid (GABA), L-glutamic acid, L-aspartic acid, glycine, acetylcholine, and ascorbic acid (final concentration 10 $\mathrm{mM}$ for each compound, $n=3$ ) were negligibly small, showing that these compounds did not interfere with the quantification of D-glucose.

To examine the stability of bGOD disks, the prepared bGOD disks were kept at $4^{\circ} \mathrm{C}$ for 1 to 5 days, and the response of each bGOD disk to $5.0 \mathrm{mM}$ D-glucose was monitored every day. The flux-time profiles with bGOD-disks kept for 5 days were practically overlapped, as shown in Fig. 4, showing that the enzymatic activity of bGOD on the avidin disk was retained for 5 days.

Application of bGOD disks to imaging of D-glucose fluxes in acute brain slices

By applying bGOD disks, the distribution of D-glucose in acute mouse hippocampal slices under the hypoxia condition was imaged. Since the acute slices were washed with a glucosefree recovery solution containing DA-64 prior to measurements, glucose in the extracellular fluids was partly washed out. The hippocampal slice on a bGOD disk was stimulated with a 50- $\mu 1$ portion of a glucose-free hypoxia solution. The flux-time profiles obtained for different neuronal regions are shown in Fig. 5A, together with two-dimensional images of the D-glucose fluxes (Fig. 6). In the CA3 and DG regions, the glucose flux 


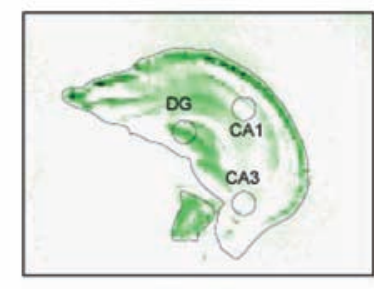

0 min

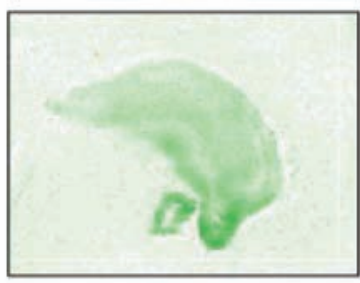

$9 \min$

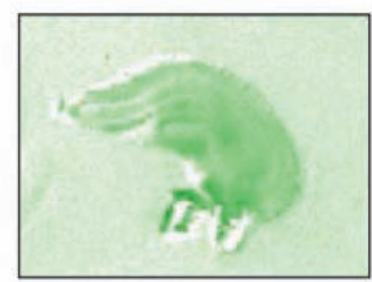

$3 \mathrm{~min}$

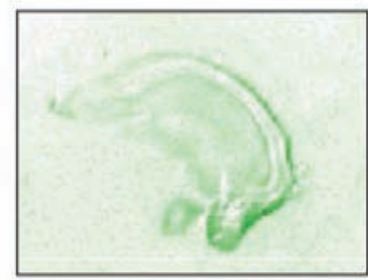

$12 \min$

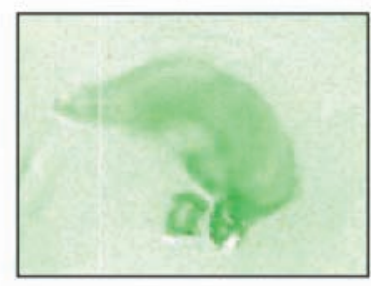

$6 \mathrm{~min}$

Fig. 6 Two-dimensional image of D-glucose fluxes in a mouse hippocampal slice under hypoxia. The image was prepared from data A in Fig. 5.

(a) DG

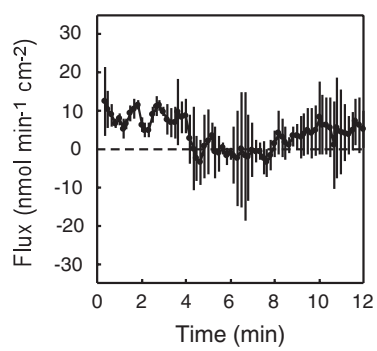

(b) $\mathrm{CA} 1$

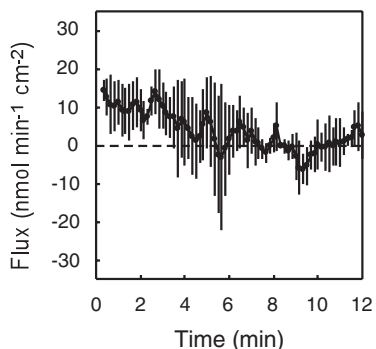

(c) $\mathrm{CA} 3$

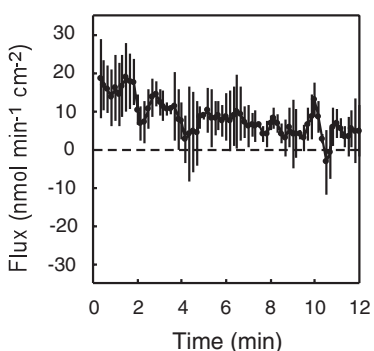

Fig. 7 Imaging of D-glucose fluxes in an acute mouse hippocampal slice stimulated by a $0.10 \mathrm{M}$ $\mathrm{KCl}$ solution. The slice was washed three times with a glucose-free recovery solution and stimulated with a $0.10 \mathrm{M} \mathrm{KCl}$ solution. The stimulation was started at $t=0$. The measurements were made at $32 \pm 2{ }^{\circ} \mathrm{C}$. The average of three measurements was plotted.

became maximum 2 and 4 min after the onset of hypoxia. Then, the fluxes gradually decreased for $10 \mathrm{~min}$, but after 10 min the fluxes increased again. For the CA1 region, no notable changes in the fluxes were observed for $10 \mathrm{~min}$. In a control, where a glucose free-recovery solution was placed instead of a hypoxia solution (case B in Fig. 5), the release of D-glucose was not observed for all of the neuronal regions. The stimulation was performed with a hypoxia (low oxygen) solution, but the solution was kept open to air during the measurements, and hence the minimum amount of oxygen required for the enzyme reaction may be supplied from air. It is known that the CA1 region is highly vulnerable and the CA3 region is more resistant to ischemic injury. ${ }^{18-20}$ The high level of L-glutamate fluxes is seen at the both regions. ${ }^{16}$ The very low flux of D-glucose at the CA1 region may be associated with ischemic injury of the CA1 region.

Similarly, the $\mathrm{KCl}(0.10 \mathrm{M})$-stimulated release of D-glucose was imaged. The flux-time profile is shown in Fig. 7. In this case, the neuronal regions, i.e., DG, CA3 and CA1, behaved similarly and the fluxes decreased monotonously. These results are in contrast to the above hypoxia case, demonstrating that the present approach is useful for the in vitro imaging of D-glucose fluxes under various types of chemical stimulation.

\section{Conclusions}

The bGOD-immobilized glass disks are useful for visualizing Dglucose fluxes at various neuronal regions of an acute brain slice. Although the method detects a flux of D-glucose in extracellular fluids, rather than intracellular glucose, and the washout of D-glucose is necessary prior to any measurements, the obtained results may be related to the neuronal activity in the hippocampal slices.

\section{Acknowledgements}

This work was supported by a Grant-in-Aid for Scientific Research from the Ministry of Education, Culture, Sports, Science and Technology, Japan. Financial support from Research Grant by College of Humanities and Sciences, Nihon University is also acknowledged.

\section{References}

1. C.-P. Chih, P. Lipton, and E. L. Roberts, Jr., Tre. Neurosci., 
2001, 24, 573.

2. J. Ai and A. Baker, Exp. Brain Res., 2006, 169, 126.

3. C. P. Chih and E. L. Roberts, Jr., J. Cereb. Blood Flow Metab., 2003, 23, 1263.

4. R. G. Shulman, D. L. Rothman, K. L. Behar, and F. Hyder, Trends Neurosci., 2004, 27, 489.

5. N. R. Sibson, A. Dhankhar, G. F. Mason, D. L. Rothman, K. L. Behar, and R. G. Shulman, Proc. Natl. Acad. Sci. U. S. A., 1998, 95, 316.

6. D. Attwell and C. Iadecola, Tre. Neurosci., 2002, 25, 621.

7. M. Ogawa, H. Watabe, N. Teramoto, Y. Miyake, T. Hayashi, H. Iida, T. Murata, and Y. Magata, Neurosci. Res., 2005, 52, 357.

8. N. Omata, T. Murata, Y. Fujibayashi, A. Waki, N. Sadato, M. Yoshimoto, Y. Wada, and Y. Yonekura, J. Cereb. Blood Flow Metab., 2000, 20, 350.

9. T. Murata, N. Omata, Y. Fujibayashi, A. Waki, N. Sadato, S. Yoshida, R. Yano, M. Yoshimoto, and Y. Yonekura, J. Neural Transmission, 1999, 106, 1075.

10. L. Pellerin and P. J. Magistretti, Proc. Natl. Acad. Sci. U. S. A., 1994, 91, 10625.
11. T. Kitano, N. Nisimaru, E. Shibata, H. Iwasaka, T Noguchi, and K. Yamada, Life Sci., 2002, 72, 557.

12. A. S. Obeidat and R. D. Andrew, Eur. J. Neurosci., 1998, 10,3451 .

13. I. Joshi and R. D. Andrew, J. Neurophysiol., 2001, 85, 414.

14. R. D. Andrew and B. A. Macvicar, Neuroscience, 1994, 62, 371.

15. N. R. Kreisman, J. C. LaManna, S.-C. Liao, E. R. Yeh, and J. R. Alcala, Brain Res., 1995, 693, 179.

16. A. Hirano, N. Moridera, M. Akashi, M. Saito, and M. Sugawara, Anal. Chem., 2003, 75, 3775.

17. A. Hirano, M. Asakawa, N. Kido, and M. Sugawara, Anal. Sci., 2000, 16, 25.

18. P. G. Aitken, G. C. Tombaught, D. A. Turner, and G. G. Somjen, J. Neurophysiol., 1998, 80, 1514.

19. N. R. Kreisman, S. Soliman, and D. Gozal, J. Neurophysiol., 2000, 83, 1031.

20. K. Shimazaki, T. Nakamura, K. Nakamura, K. Oguro, T. Masuzawa, Y. Kudo, and N. Kawai, NeuroReport, 1998, 9, 1875 . 